\title{
Characterization of pectin methylesterase gene family and its possible role in juice sac granulation in navel orange (Citrus sinensis Osbeck)
}

\author{
Zixuan $\mathrm{Li}^{1,2 \dagger}{ }^{2}$, Liming Wu ${ }^{1 \dagger}$, Ce Wang ${ }^{1}$, Yue Wang ${ }^{2}$, Ligang He${ }^{1}$, Zhijing Wang ${ }^{1}$, Xiaofang Ma ${ }^{1}$, Fuxi Bai ${ }^{1}$,
} Guizhi Feng ${ }^{2}$, Jihong Liu ${ }^{2}$, Yingchun Jiang ${ }^{1^{*}}$ and Fang Song ${ }^{1^{*}}$

\begin{abstract}
Background: Citrus is one of the most important fresh fruit crops worldwide. Juice sac granulation is a physiological disorder, which leads to a reduction in soluble solid concentration, total sugar, and titratable acidity of citrus fruits. Pectin methylesterase (PME) catalyzes the de-methylesterification of homogalacturonans and plays crucial roles in cell wall modification during plant development and fruit ripening. Although PME family has been well investigated in various model plants, little is known regarding the evolutionary property and biological function of PME family genes in citrus.

Results: In this study, 53 non-redundant PME genes were identified from Citrus sinensis genome, and these PME genes were divided into four clades based on the phylogenetic relationship. Subsequently, bioinformatics analyses of gene structure, conserved domain, chromosome localization, gene duplication, and collinearity were performed on CSPME genes, providing important clues for further research on the functions of CSPME genes. The expression profiles of CSPME genes in response to juice sac granulation and low-temperature stress revealed that CSPME genes were involved in the low temperature-induced juice sac granulation in navel orange fruits. Subcellular localization analysis suggested that CSPME genes were localized on the apoplast, endoplasmic reticulum, plasma membrane, and vacuole membrane. Moreover, yeast one-hybrid screening and dual luciferase activity assay revealed that the transcription factor CSRVE1 directly bound to the promoter of CSPME3 and activated its activity.
\end{abstract}

Conclusion: In summary, this study conducts a comprehensive analysis of the PME gene family in citrus, and provides a novel insight into the biological functions and regulation patterns of CsPME genes during juice sac granulation of citrus.

Keywords: Citrus sinensis, Pectin methylesterases (PMEs), Juice sac granulation, Transcription factor

*Correspondence: hbcitrus@126.com; fsong_ray@163.com

†Zixuan Li and Liming Wu contributed equally to this work.

${ }^{1}$ Institute of Fruit and Tea, Hubei Academy of Agricultural Sciences,

Wuhan 430064, PR China

Full list of author information is available at the end of the article

\section{Background}

The plant cells are surrounded by a rigid extracellular structure called cell wall which is composed of polysaccharides (cellulose, hemicelluloses, and pectin), phenolics, and cell wall proteins [1, 2]. Pectin, the major constituent of the plant cell wall, is a heteropolysaccharides containing homogalacturonan (HG), 
rhamnogalacturonan-I (RG-I), rhamnogalacturonan II (RG-II), and xylogalacturonan (XGA) components [3, 4]. HG, a linear homopolymer of a-1,4-linked galacturonic acid, is the dominant form of pectic polysaccharide, accounting for $\sim 65 \%$ of total pectin in plant cells [5]. HG is synthesized in Golgi apparatus and delivered to the apoplast in a highly methylesterified state [6].

Pectin methylesterase (PME), also known as pectinesterase, catalyzes the removal of the methyl group from HG, thus generating methanol and negatively charged carboxyl groups in the de-methylesterification process [7]. The de-methylesterified HG is often susceptible to be degraded by pectin-degrading enzymes including polygalacturonases and pectate lyases, which may loosen the cell wall [8-10]. Alternatively, when the degradation process is blocked, de-methylesterified HG is able to cross-link with calcium ions to form a structure called "egg box", which may rigidify the cell wall $[11,12]$. The extent of HG de-methylesterification may be affected by PME inhibitors (PMEIs) which directly bind to the catalytic domain of PMEs to inhibit their enzyme activity $[7,11]$. According to the protein structure, the PMEs fall two groups, namely, Type-I PMEs (with PME domain and an additional $\mathrm{N}$-terminal pro-region similar to PMEI domain) and Type-II PMEs (with PME domain only) [13]. PME is widely present in higher plants. Genomewide identification of $P M E$ gene family has been reported in many plant species. Specifically, 66 PME genes were identified from Arabidopsis thaliana [14], 110 from Brassica rapa [15], 80 from Gossypium arboretum [16], 43 from Oryza sativa [17], 105 from Linum usitatissimum [18], and 57 from Lycopersicon esculentum [19]. Previous studies have revealed that $P M E$ genes exert various functions in plants, such as fruit ripening [20,21], fruit softening [22], pollen development and growth [23, 24], root hair formation, seed germination [25], as well as biotic and abiotic stress response [26-29].

Citrus, as one of the most important fresh fruit crops worldwide, is valued for its high levels of functional components, including volatiles [30], flavonoids [31, 32], carotenoids [33, 34], and polysaccharides [35]. However, the juice sac granulation is a serious physiological disorder that often occurs in the on-tree fruits of late-ripening citrus cultivars and postharvest fruits of normal citrus cultivars during storage [36, 37]. Granulation leads to a great reduction in soluble solid concentration, total sugar, and titratable acidity, thus decreasing the edible value and commercial value of citrus fruits [36, 38, 39]. Previous studies have suggested that pectin is significantly accumulated in granulated citrus fruits, and the expression profiles of $P M E$ genes are significantly changed during granulation, indicating a strong association of $P M E$ genes with citrus fruit granulation [37, 39]. However, the knowledge of $P M E$ family genes in citrus remains very limited.

In this study, a genome-wide analysis was performed to identify the whole PME family genes in Citrus sinensis. The phylogenetic relationship, gene structure, conserved domain, chromosomal localization, gene duplication events and collinear correlation of CsPME family genes were analyzed. Furthermore, the spatial and temporal expression patterns of CsPME genes and their expression profiles in response to juice sac granulation and low-temperature treatments were assessed. Moreover, the yeast one-hybrid screening and dual luciferase activity assay were performed to investigate the regulation network of CsPME genes during juice sac granulation. Our results extend the knowledge of citrus $P M E$ genes and provide an important basis for further research on their functions during juice sac granulation of citrus fruits.

\section{Results}

\section{Genome-wide identification of PME family genes from Citrus sinensis}

To identify PME family genes from C. sinensis, HMMER and BLASTP analyses of the sweet orange genome (C. sinensis, version 2) were performed [40]. The predicted CsPME proteins containing both PME and PMEI domains were designated as Type-1 PMEs, and the proteins containing only PME domain (but no PMEI domain) were designated as Type-2 PMEs. After verification with CDD and SMART, a total of 53 non-redundant CsPME proteins were identified from $C$. sinensis genome, including 29 Type-I and 25 Type-II (Supplementary Table S1). These 53 CsPME genes were named according to their physical location on the chromosomes. Basic information on CsPME genes, including their gene name, locus ID, number of amino acid (aa) residues, molecular weight $(\mathrm{Da})$, isoelectric point $(\mathrm{pI})$, grand average of hydropathicity (GRAVY), instability index (II), and gene position, was shown in Supplementary Table S2. The CsPME proteins varied from 144 to 632 aa in length with a predicted molecular weight ranging from $16,814.69$ to 69,600.03 $\mathrm{Da}$ and a theoretical isoelectric point (pI) from 4.68 to 10.69 .

\section{Phylogenetic analysis of CsPME genes}

To explore the phylogenetic relationship of CsPMEs between citrus and Arabidopsis, a phylogenetic tree was constructed with the amino acid sequences of 53 CsPMEs and 66 AtPMEs by using the Maximum Likelihood Method of MEGA X. As shown in Fig. 1, the PME family was clustered into 4 clades according to the previous definition of AtPMEs [14]. The Type-I CsPMEs (with a PMEI domain) were clustered into Clade II, Clade III, 


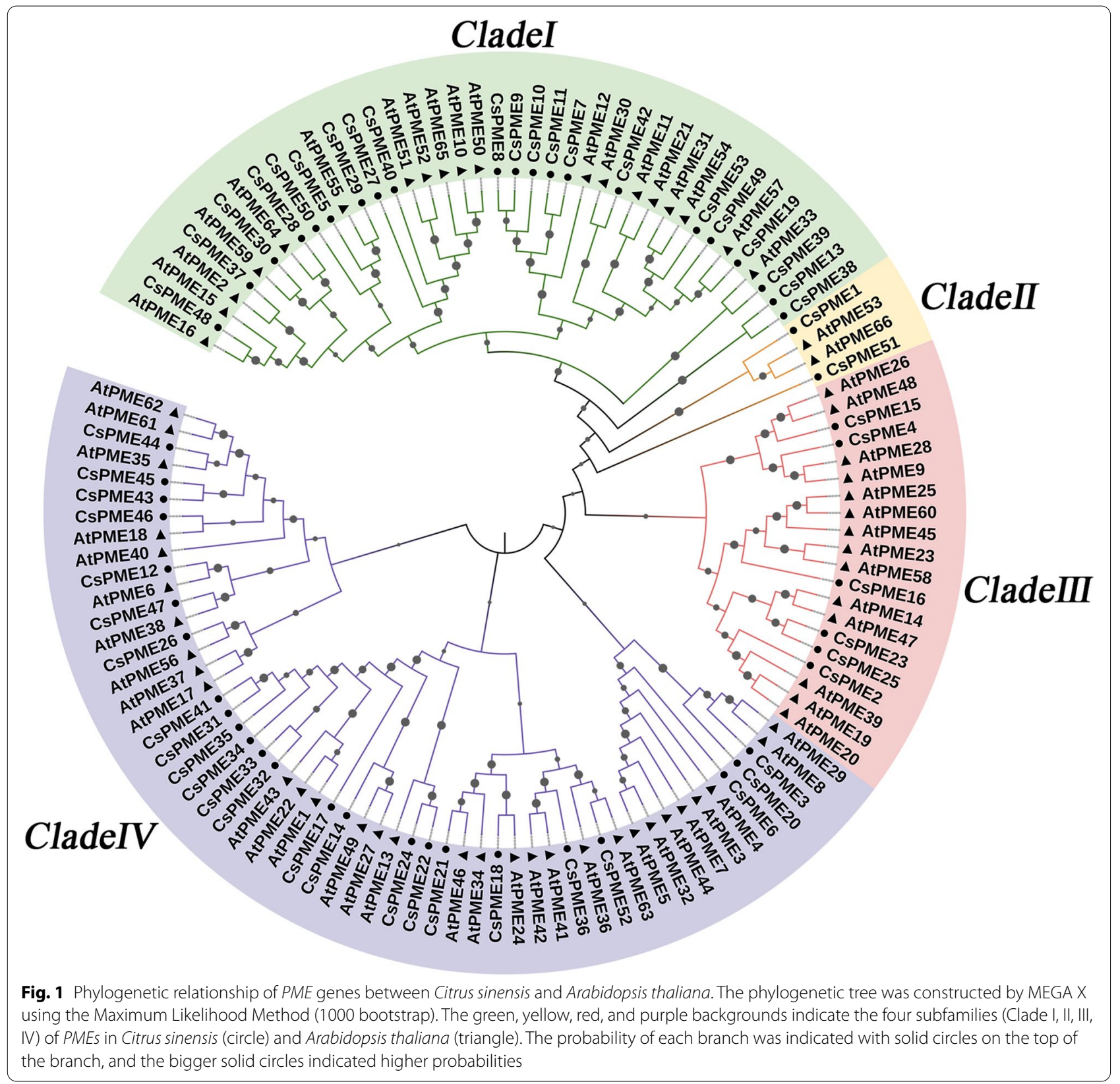

and Clade IV, whereas Type-II CsPMEs (without a PMEI domain) were mainly clustered into Clade I.

\section{Conserved motif and gene structure analyses of CsPME genes}

The conserved motifs were analyzed based on the coding sequences of CsPMEs. As shown in Fig. 2a, a total of 15 conserved motifs were predicted in CsPME proteins using MEME website, and the conserved protein sequences were displayed in Fig. 2c, and the size of the motifs ranged from 15 (motif 9) to 50 aa (motif 1 ). Most $P M E$ members in the same clade shared similar conserved motifs. Based on the InterPro database, motif $1,2,3,4,5,6$, and 7 were annotated as pectinesterase activity domains. Motif $1,2,3,4,5,6$, and 7 were present in $49,47,46,47,48,50$, and 48 CsPME proteins, indicating that these pectinesterase-related motifs were highly conserved in CsPME proteins. Most interestingly, motif 10 was annotated to encode the PMEI domain, which was in line with the result that motif 10 was presented in 29 Type-I CsPME proteins. 


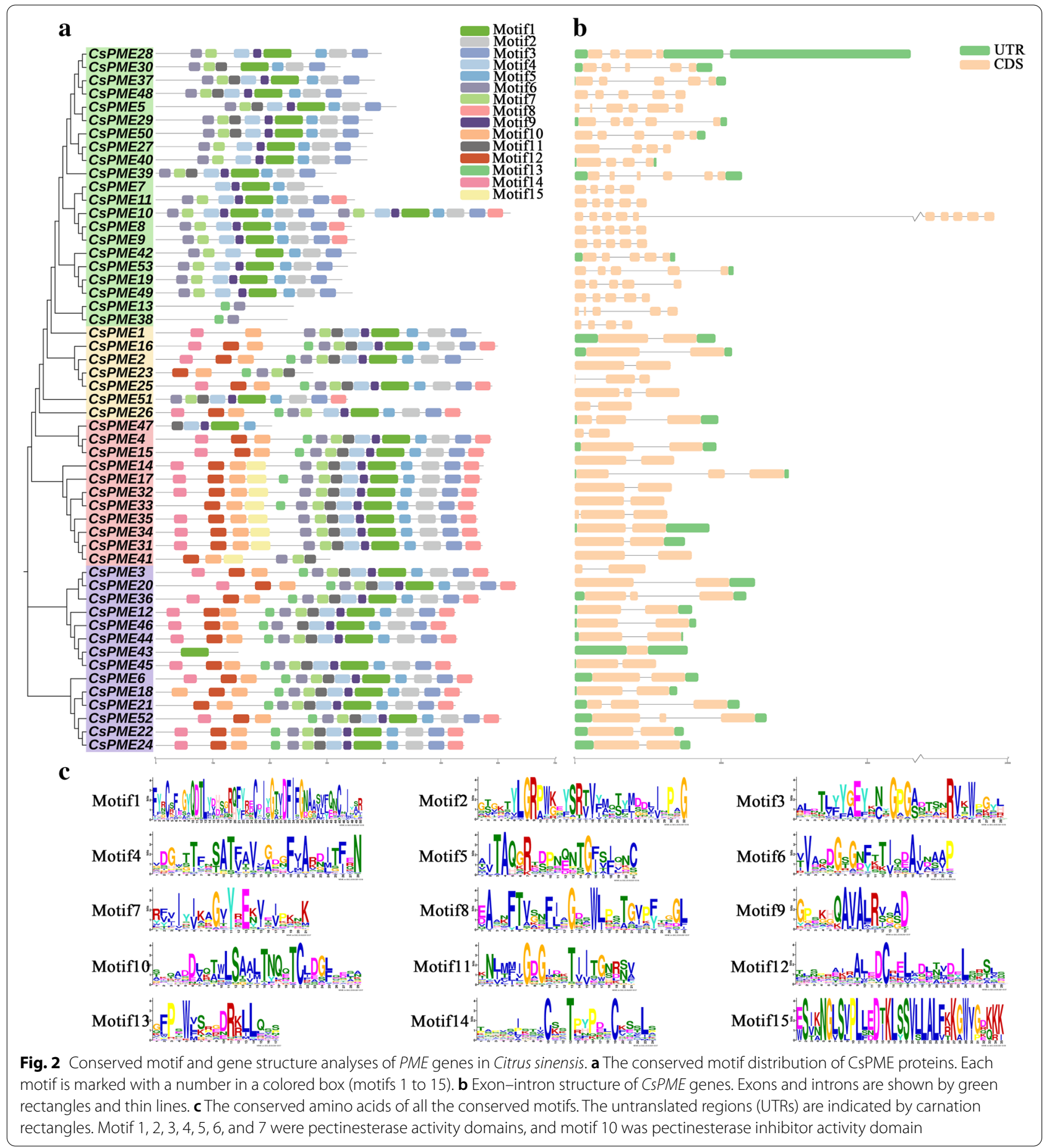

To further characterize the gene structural diversity of $P M E$ family in sweet orange, the exon-intron organizations were analyzed based on the genome sequence of CSPME genes. The number of exons varied from 2 to 10 . The gene structure analysis also revealed that CsPME genes in the same clade usually shared similar gene structures. As shown in Fig. 2b, the exon numbers of Type-II CsPME genes were relatively larger than that of Type-I CsPME genes, but their exon sizes were relatively smaller. The conservation of both conserved motif and gene structure of CsPME family members in the same clade strongly supported the reliability of the phylogenetic classification in Fig. 1. 


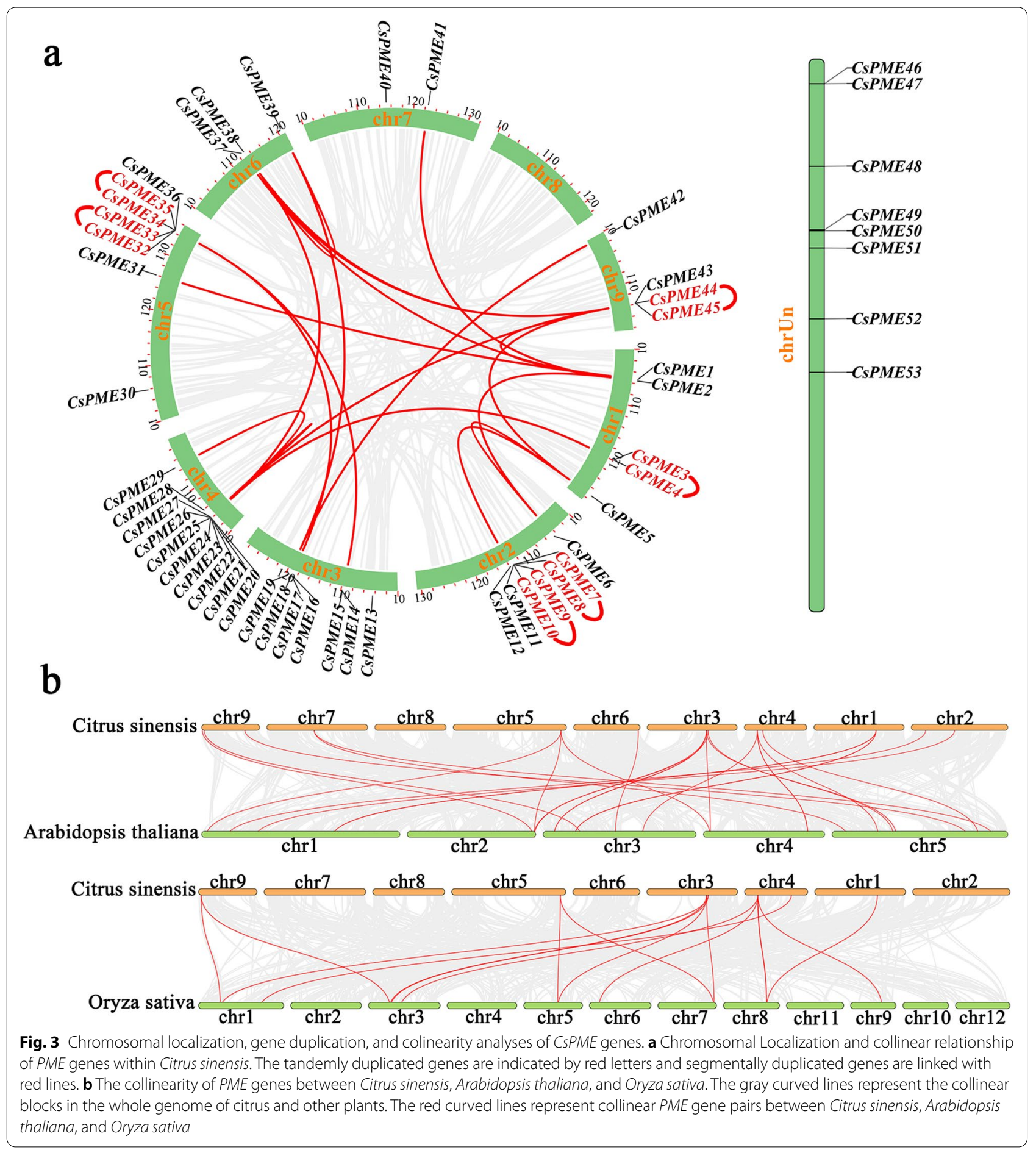

\section{Chromosomal localization, gene duplication, and synteny} analysis

Genome chromosomal localization analysis revealed that $53 C s P M E$ genes were distributed on 8 chromosomes except Chromosome 8 (Fig. 3a). Chr 4 had the largest number (10) of CsPME genes, follow by Chr 2 (7) and Chr 5 (7). Additionally, 8 CsPME genes were failed to be localized on the total 9 chromosomes. Gene duplication provided raw materials for generating new genes and functions. The gene duplication analysis of CsPMEs revealed that 6 pairs of $C s P M E$ genes (corresponding to 12 CsPME genes) were identified as tandemly duplicated 
genes, and 17 pairs of CsPME genes (corresponding to 19 CsPME genes) were identified as segmentally duplicated genes (Fig. 3a). These results indicated that segmental duplication might contribute more to the expansion of CsPME genes compared to tandem duplication.

To further identify the orthologous genes of CsPMEs as well as their evolution relationship, two comparative syntenic relationship was established based on two representative species, $A$. thaliana (dicot) and $O$. sativa (monocot). As shown in Fig. 3b, a total of 27 orthologous $P M E$ gene pairs were identified between citrus and Arabidopsis, and 16 orthologous $P M E$ gene pairs were identified between citrus and rice. Interestingly, some orthologous $P M E$ gene pairs identified between citrus and rice were not detected between citrus and Arabidopsis, such as CsPME22/OsPME21, CsPME25/OsPME29, and CsPME29/OsPME7, suggesting that these orthologous gene pairs were generated after the divergence of dicotyledonous and monocotyledonous plants. Additionally, one citrus $P M E$ gene usually matched three or more Arabidopsis $P M E$ genes. For example, CsPME3 was orthologous to AtPME8, AtPME9, AtPME28, and AtPME29, and CsPME32 to AtPME1, AtPME22, and AtPME43, which implied that these genes in A. thaliana were paralogous gene pairs.

\section{Spatial and temporal expression patterns of CsPME genes in citrus fruits}

To further assess the spatial-temporal expression profiles of CsPME genes in navel orange fruits, the transcriptome data of 4 fruit tissues (namely, epicarp, albedo, segment membrane, and juice sac) at 6 development stages (namely, 0 DAF, 80 DAF, 120 DAF, 155 DAF, 180 DAF, and 220 DAF) of 'Fengjie' navel orange from our previous study were adopted (Supplementary Table S3) [41]. As shown in Fig. 4, of 53 CsPME family genes, 34 CsPMEs were expressed in navel orange fruits, indicating an indispensable role of CsPME genes in citrus fruits. CsPME3, CsPME39, CsPME4, and CsPME52 were highly expressed in all the 4 fruit tissues. CsPME20 and CsPME36 were highly expressed in juice sacs and segment membranes. CsPME6 and CsPME16 were highly expressed in juice sacs. CsPME26 and CsPME22 were highly expressed in epicarps. Additionally, the expression levels of CsPME3, CsPME4, CsPME20, CsPME21, and CsPME53 were decreased from 50 to $120 \mathrm{DAF}$, and then increased at 155 DAF, followed by a decrease until 220 DAF. The expression level of CsPME39 was increased from 50 to 120 DAF, and then decreased from 155 to $180 \mathrm{DAF}$, followed by an increase at 220 DAF. The expression level of CsPME16 was increased throughout the development stages, while CsPME26 and CsPME37 were decreased. In summary, these results indicated that CsPME genes might play significant roles in navel orange fruits development.

\section{Expression patterns of CSPME genes at different granulation levels of 'lane late' navel orange fruits}

To explore the functions of CsPME genes in juice sac granulation, we investigated the expression patterns of CsPME genes in 'Lane late' navel orange fruits at different granulation levels, including non-granulation (CK), slight granulation (GR1), moderate granulation (GR2), and serious granulation (GR3), by using transcriptome sequencing (Fig. 5a, Supplementary Table S4). As a result, 11 CsPME genes were differentially expressed in comparison of granulated samples and normal samples (Fig. 5b). Except for the down-regulation of CsPME6, other 10 differentially expressed CsPME genes were up-regulated in granulated fruit samples, indicating these CsPMEs might be involved in juice sac granulation of navel orange. Further, qRT-PCR was utilized to validate their expression profiles. As shown in Fig. 5c, most CsPMEs exhibited the same expression patterns as those in transcriptome data., but the expression pattern of CsPME6 was opposite between transcriptome data and qRT-PCR at GR3 level. Most interestingly, CsPME1, CsPME3, and CsPME6 were induced at the first granulation level (GR1), indicating these three CsPMEs might be involved in the initiation of juice sac granulation. In addition, we also assessed the expression patterns of other cell wall modification related genes during juice sac granulation of navel orange. As shown in Fig. S1, CsPAL (phenylalanine ammonia lyase), CsCAD (cinnamyl alcohol dehydrogenase), CsPOD (peroxidase), and CsPG (polygalacturonase) were induced in the granulated navel orange fruits, which further demonstrated that juice sac granulation progress was accompany with cell wall modifications.

\section{Expression patterns of CsPME genes under low temperature treatment}

Our previous study had revealed that juice sac granulation of 'Lane late' navel orange in the Three Gorges area was mainly caused by the low temperature in the winter [37]. Thus, the expression patterns of CsPMEs under low temperature treatment were investigated in this study. As shown in Fig. 6, nine granulation-induced genes were also induced by low temperature treatment. Additionally, CsPME3, CsPME20, and CsPME34 were induced at the beginning of treatment $(3 \mathrm{~h})$, indicating these three CsPMEs might be involved in the initiation of low temperature response in 'Lane late' navel orange. Notably, CsPME3 was continuously highly-induced in all treatment group at all the detection time points, which further suggested a potential significant role of CsPME3 in 


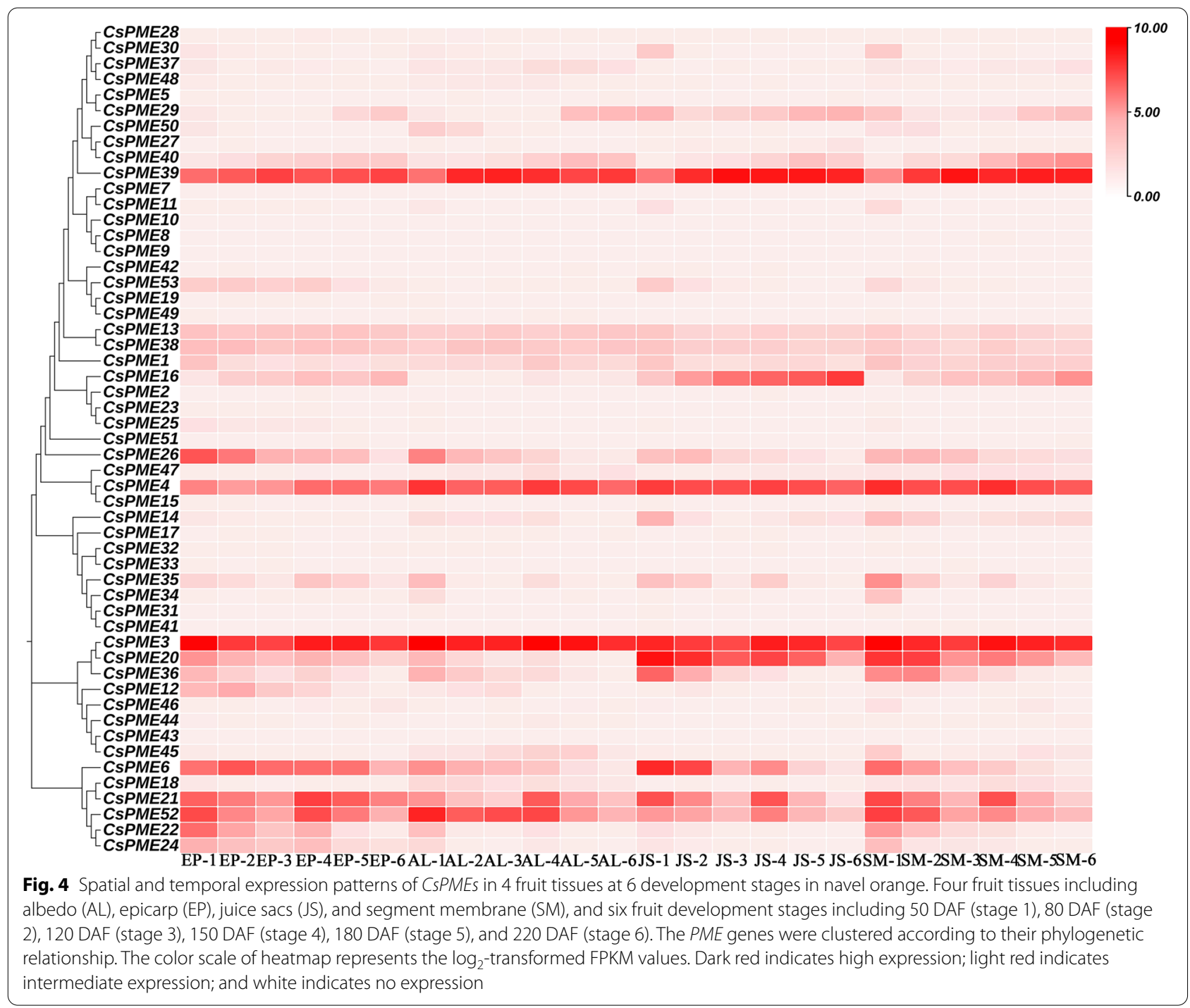

low temperature-induced juice sac granulation in 'Lane late' navel orange.

\section{Subcellular localization of CsPME genes}

To explore the subcellular localization of CsPME proteins, the coding sequence (CDS) of CsPMEs (including CsPME6, CsPME34, CsPME35, CsPME40, and CsPME52) without stop codon was ligated with that of green fluorescent protein (GFP) and cloned into pICH86988 vectors, and these vectors were co-expressed with corresponding markers in tobacco leaves. As shown in Fig. 7a, the green fluorescence of CsPME35:GFP, CsPME40:GFP, and CsPME52:GFP were overlapped with the red fluorescence of endoplasmic reticulum marker (HDEL:OFP), revealing that CsPME35, CsPME40, and CsPME52 were localized in the endoplasmic reticulum. Additionally, the green fluorescence of CsPME6:GFP, and CsPME34:GFP were co-localized with the red fluorescence of plasma membrane marker (CBL1n:OFP) and vacuole membrane marker (CBL3n:OFP), respectively (Fig. 7b, c).

According to a previous study, the weaker green fluorescence in the apoplast might be caused by the lower stability of the protein in the apoplast than that in the endoplasmic reticulum [42]. Thus, the CDS of CsPME3 and CsPME21 was further ligated with that of mCherry and cloned into pICH86988 vectors, and these vectors were co-expressed with an apoplast marker RAmy3A:GFP. As shown in Fig. 7d, the red fluorescence of CsPME3:mCherry and CsPME21:mCherry was respectively co-localized with the green fluorescence of RAmy3A:GFP, indicating that CsPME3 and CsPME21 were localized in the cell wall. Based on these, we hypothesized that CsPME3 and CsPME21 might function as pectin-degrading enzymes in cell wall, and they might be 


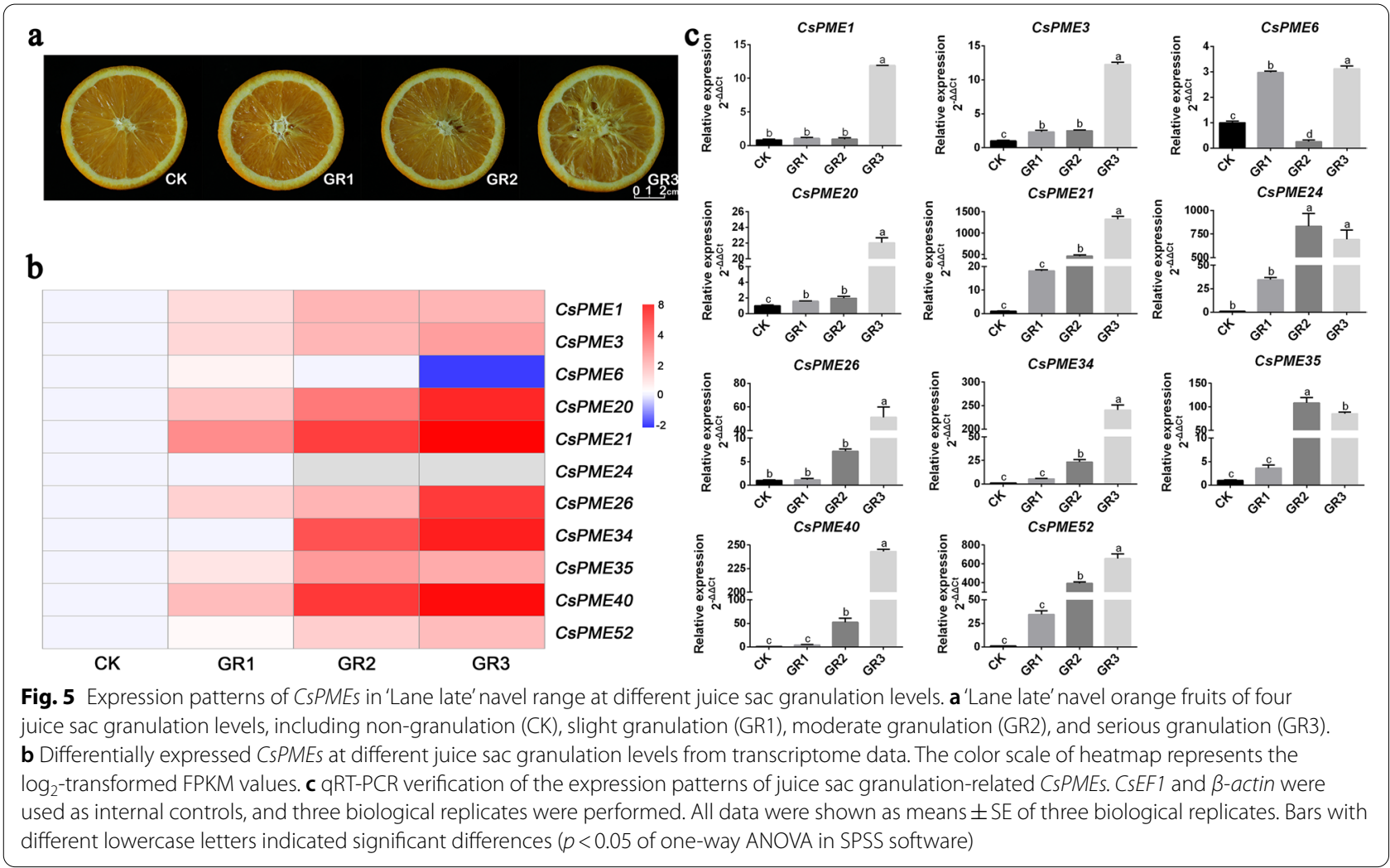

involved in the low temperature-induced juice sac granulation of navel orange.

\section{Identification of interactive transcription factor of proCsPME3}

Due to the important role of CSPME3 in low temperature-induced juice sac granulation, we further explored the regulation network of CsPME3. The $2 \mathrm{~kb}$ upstream sequence of CsPME3 was cloned as promoter sequence, and the cis-elements of proCsPME3 was analyzed using PlantCARE online tool. As shown in Fig. S2, cis-elements related to plant hormones (ABRE, TGACG-motif, CGTCA-motif, TCA-element, and TGA-element) and biotic/abiotic stress response (TC-rich repeats) were detected in the promoter of CsPME3. Interestingly, MYB recognition site was also found in the proCsPME3, indicating that CsPME3 might be regulated by MYB transcription factors. To further identify the regulatory transcription factor of $C s P M E 3$, the promoter sequence of CsPME3 was ligated into pAbAi to construct bait vector. Then, the bait vector was utilized to screen the yeast one-hybrid $(\mathrm{Y} 1 \mathrm{H})$ library, and 8 positive clones were obtained. The point-to-point $\mathrm{Y} 1 \mathrm{H}$ assay indicated that of 8 positive clones, only the clones of CsRVE1 (Cs6g16000) + proCsPME3 were grown well on the SDleu $+200 \mathrm{ng} / \mu \mathrm{L}$ AbAi (aureobasidin A) medium (Fig. 8a), indicating that CsRVE1 directly interacted with the promoter of CsPME3. Dual luciferase transcriptional activity assay (LUC) was further performed on tobacco leaves to investigate the regulation effect of CsRVE1 on CsPME3 with $C s R V E 1$ as effector and proCsPME3 as reporter. As shown in Fig. 8b, the activity of proCsPME3 was significantly induced by CSREV1. In summary, these results suggested that the transcription factor CsREV1 was involved in low temperature-induced juice sac granulation by regulating the activity of CsPME3.

\section{Subcellular localization and expression patterns of CSRVE1}

To investigate the subcellular localization of CsRVE1, coding sequence of CsRVE1 was fused with GFP, cloned into pICH86988 vector, and co-expressed with nucleolus marker [43]. As shown in Fig. 8c, the green fluorescence of CsRVE1:GFP was co-localized with the red fluorescence of nucleolus marker (FIB2:mCherry), suggesting that CsRVE1 protein was localized to the nucleolus. Further, the spatial and temporal expression pattern of CSRVE1 during fruit development was evaluated based on our previous transcriptome data of 4 fruit tissues during 6 development stages of navel orange. As shown in Fig. 8d, CsRVE1 was highly expressed in all the 4 fruit tissues, which was in line with the expression pattern of CsPME3. In addition, the expression level of CsRVE1 was 


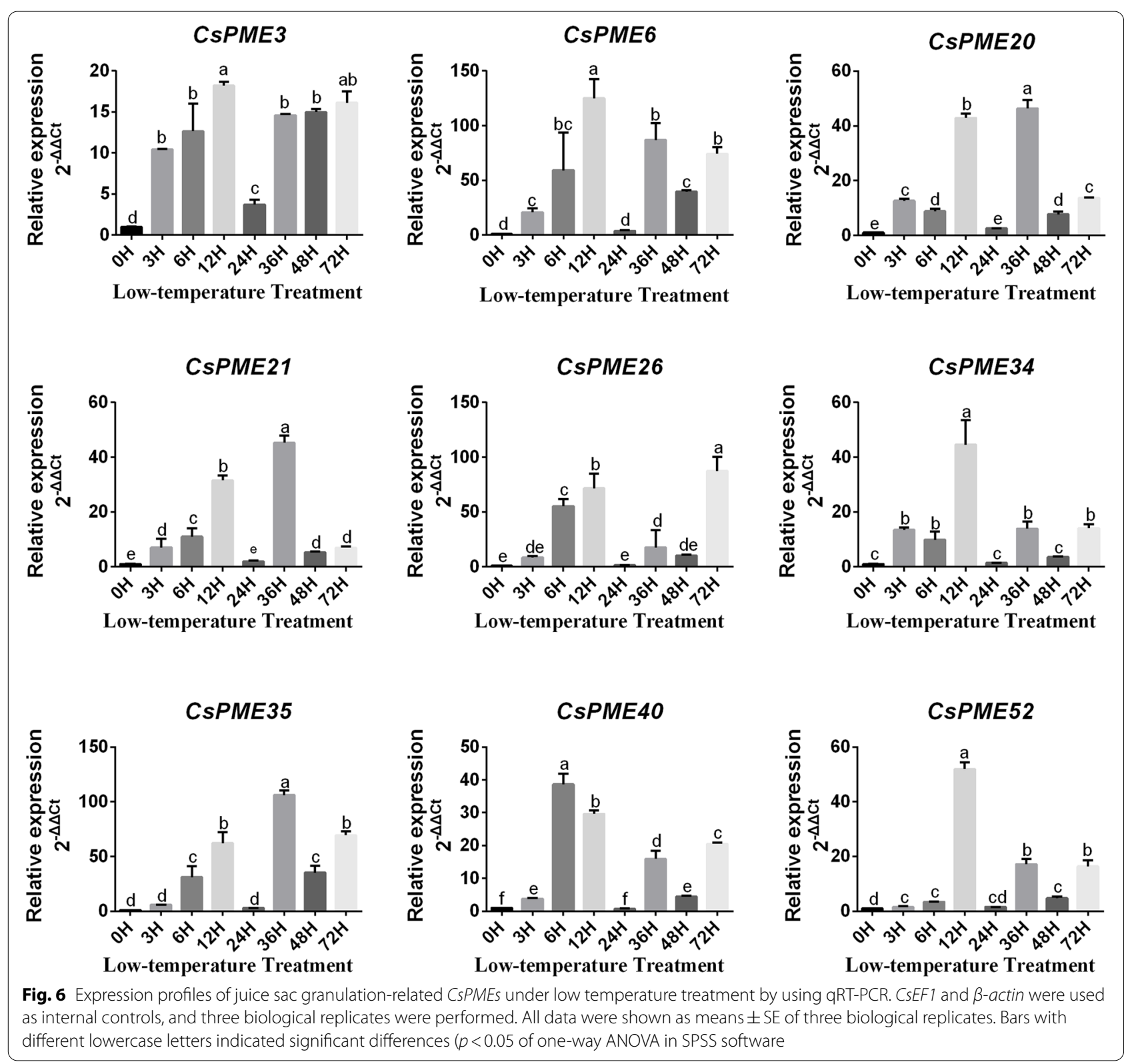

continuously increased throughout the 6 fruit development stages, indicating that CsRVE1 might be involved in the fruit ripening process. Moreover, we also found that the expression level of CsRVE1 was induced by low temperature treatment at $3 \mathrm{~h}$ and $48 \mathrm{~h}$ (Fig. 8e). Taken together, these results further implied that CsRVE1 might play an important regulatory role in low temperatureinduced juice sac granulation.

\section{Discussion}

The pectin methylesterases (PME) family plays a crucial role in fruit ripening and softening, pollen development and growth, root hair formation, seed gemination, biotic and abiotic stress response, and other developmental processes in plants [20-26]. According to its significant functions, the $P M E$ gene family was widely identified in various plant species. However, the classification and biological functions of $P M E$ gene family remains largely unclear in citrus. In this study, a total of 53 CsPME genes were identified from citrus via genome-wide analysis. The number of $P M E$ genes was larger in citrus than in O. sativa (43) [17], but smaller than in A. thaliana (66) [14], G. arboretum (80) [16], B. rapa (110) [15], and L. usitatissimum (105) [18]. The phylogenetic analysis revealed that CsPME genes were clustered into four clades, which was further verified 
by the gene structure and conserved motifs analyses. The gene duplication analysis revealed that segmental duplication events might contribute more to the expansion of CsPME genes than tandem duplication events.

The spatial and temporal expression profiles provide important clues for investigating the biological function of CsPME genes. The high-spatiotemporal-resolution transcriptome data of navel orange fruits from our previous study [41] indicated that much more CsPME family genes (around 64\%, 34 out of 53) were expressed in citrus than in tomato (27 SIPMEs) and strawberry (5 FvPMEs) during fruit development [19]. These results strongly suggested that CsPMEs might play indispensable roles in fruit development in navel orange. Some CsPME genes exhibited tissue-specific expression patterns. For example, CsPME2O and CsPME36 were highly expressed in juice sac and segment membrane, CsPME6 and CsPME16 in juice sac, and CsPME26 and CsPME22 in epicarp, suggesting that these CsPME genes might have particular function in the development of specific tissues. However, CsPME3, CsPME39, CsPME4, and CsPME52 were highly expressed in all the fruit tissues. The phylogenetic analysis demonstrated that CsPME3 was clustered together with AtPME3 (At3g14310, designed as AtPME29 in Fig. 1) which was particularly active in leaves, stems, and roots of Arabidopsis seedlings [44]. Moreover, AtPME3 was reported to play significant roles in adventitious rooting, root hypersensitivity to zinc, plant-nematode interaction, pathogen infection, and seed gemination [25, 44-47]. Thus, CsPME3, CsPME39, CsPME4, and CsPME52 might have multiple biological functions in fruits development. Additionally, the expression levels of those highly expressed CsPME genes, including CsPME3, CsPME4, CsPME20, CsPME21, and CsPME53, were decreased from 50 to $120 \mathrm{DAF}$, and then increased at $155 \mathrm{DAF}$, followed by a decrease until 220 DAF. The $155 \mathrm{DAF}$ was the late fruit expansion stage, and $220 \mathrm{DAF}$ was the fruit ripening stage [41], indicating these genes might be involved in the fruit expansion and ripening in navel orange.

Most interestingly, 11 CsPME genes were found to be differentially expressed in comparison of granulated fruits and non-granulated fruits in both transcriptome data and qRT-PCR results of navel orange, indicating that these
CsPME genes might be related to the juice sac granulation of 'Lane late' navel orange. Additionally, $10 \mathrm{Cs} P M E$ genes were induced in the granulated fruits, which was consistent with our previous study of navel orange [37], but disagreed with the findings in Ponkan mandarin [39]. We deduced that this variation could be attributed to the different cell wall metabolism between tight skin and loose skin varieties of citrus [48]. Moreover, CsPME1, CsPME3, and CsPME6 were induced at the first granulation level (GR1) with less than $25 \%$ granulation area of total fruit (Fig. 5b), indicating that these genes might be involved in the initiation of juice sac granulation. Our previous study reported that the juice sac granulation of 'Lane late' navel orange was mainly due to the low temperature in the winter [37], and this study found that 9 juice sac granulationrelated CSPME genes were induced by low temperature treatment, especially at $6 \mathrm{~h}$, which was in line with previous studies results that $P M E$ family genes were up-regulated by cold stress in B.napus and A. thaliana [49-51]. It should be noted that CsPME3 was consecutively highlyexpressed at all the detection time points, which further suggested that CsPME3 might be involved in the initiation of low temperature-induced juice sac granulation in 'Lane late' navel orange.

The subcellular localization analysis revealed that three CsPME proteins were localized on the endoplasmic reticulum, one on plasma membrane, one on vacuole membrane and two on the apoplast, which strongly supported the hypothesis that CsPME genes played multiple biological functions in citrus fruits. Most interestingly, CsPME3 and CsPME21 were localized on the apoplast (cell wall), which was in accordance with the findings of its orthologous genes, AtPME3 and FvPME39, in Arabidopsis and strawberry, respectively [19, 44]. Additionally, AtPME3 was ubiquitously expressed in $A$. thaliana, particularly in vascular tissues, and AtPME3 was functioned as a pectin-degrading enzyme during the cell wall remodeling process $[25,44,52]$. FvPME39 was identified as pectinmodifying enzyme to regulate fruit firmness, pectin content, and cell wall structure during fruit softening [19]. The above-mentioned findings indicated that CsPME3, as a pectin-modifying enzyme, might play significant roles in cell wall structure modification and fruit rigidification during juice sac granulation in 'Lane late' navel orange.

(See figure on next page.)

Fig. 7 Subcellular localization of CsPME proteins in tobacco leaves. a The green fluorescence of CsPME35:GFP, CsPME40:GFP, and CsPME52:GFP was overlapped with the red fluorescence of endoplasmic reticulum marker (HDEL:OFP), respectively. $\mathbf{b}$ The green fluorescence of CsPME6:GFP was co-localized with the red fluorescence of plasma membrane marker (CBL1n:OFP). c The green fluorescence of CsPME34:GFP was co-localized with the red fluorescence of vacuole membrane marker (CBL3n:OFP). $\mathbf{d}$ The red fluorescence of CsPME3:mCherry and CsPME21:mCherry was co-localized with the green fluorescence of RAmy3A:GFP, respectively. CSPME6, CSPME34, CsPME35, CSPME40, and CsPME52 were fused with GFP protein. CSPME3 and CSPME21 were fused with mCherry protein. ER-marker (HDEL:OFP), apoplast marker (RAmy3A:GFP), plasma membrane marker (CBL1n:OFP), and vacuole membrane marker (CBL3n:OFP) were utilized for co-localization analysis and imaging 


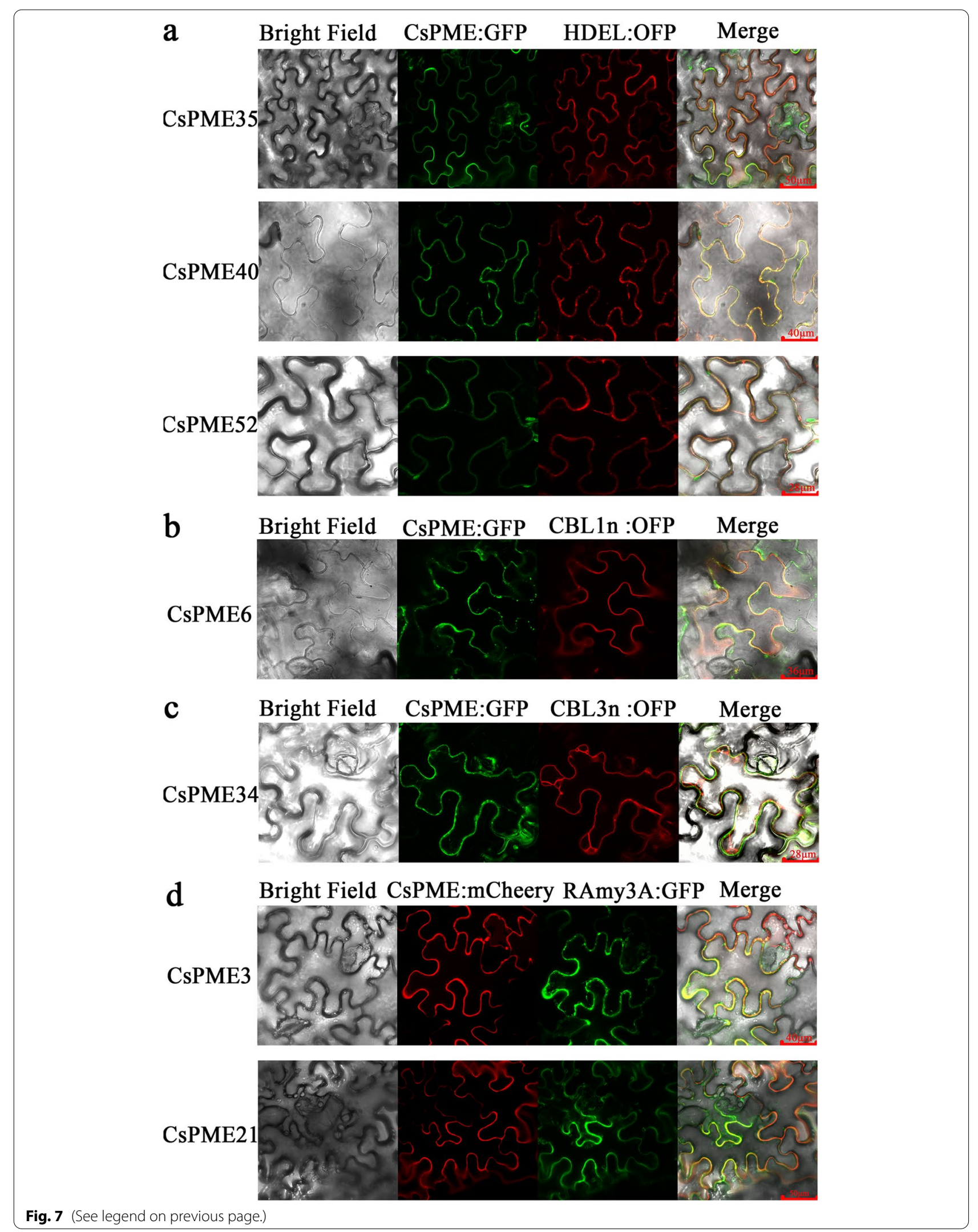



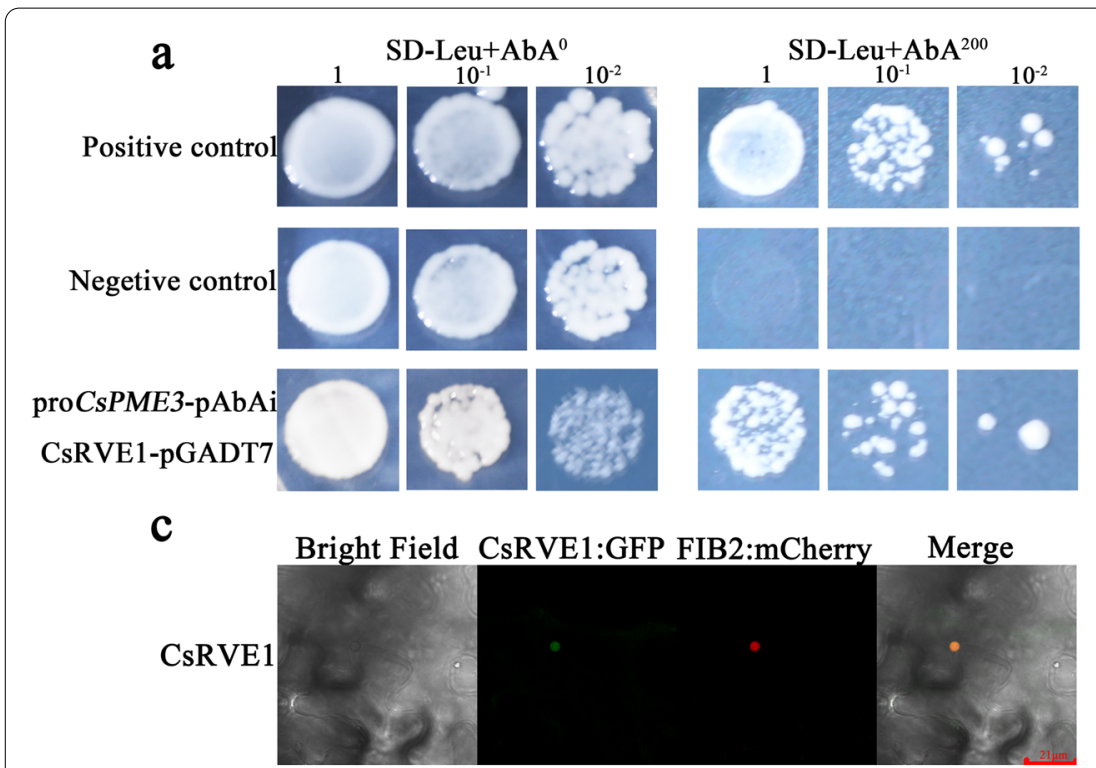

d

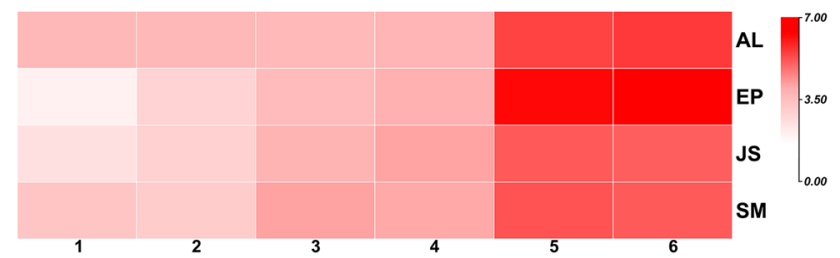

b Effector
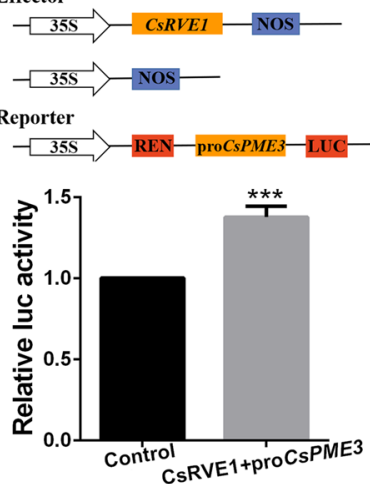

e

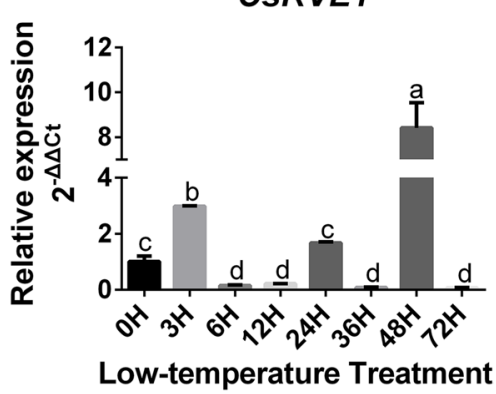

Fig. 8 Transcription factor CSRVE1 bound to the promoter of CSPME3 and activated its activity. a Yeast one-hybrid assay suggested the interaction between CSRVE1 and the promoter of CSPME3. b Dual luciferase transcriptional activity assay revealed the activation of proCSPME3 by CSRVE1. $\mathbf{C}$ Subcellular localization of CSRVE1 in tobacco leaves. CSRVE1 was fused with GFP, and nucleolus marker (FIB2:mCherry) was utilized for co-localization analysis and imaging. $\mathbf{d}$. Spatial and temporal expression profiles of CSRVE1 in navel orange fruits. The color scale of heatmap represents the $\log _{2}$-transformed FPKM values. e. Expression patterns of CSRVE1 under low temperature stress. CSEF1 was used as internal control, and three biological replicates were performed. Three individual biological replicates were performed for Dual luciferase transcriptional activity assay and qRT-PCR, data were shown as means \pm SE of three biological replicates, and bars with different lowercase letters indicated significant differences $(p<0.05$ of one-way ANOVA in SPSS software)

To further reveal the regulation mechanism of CsPME3 during juice sac granulation, the promoter sequence of CsPME3 was utilized as a bait to screen a yeast one-hybrid library. The transcription factor CsRVE1 was found to directly interact with the proCsPME3. Additionally, LUC assay further illustrated that CsRVE1 activated the activity of proCsPME3, and the subcellular localization analysis showed that $C s R V E 1$ was localized to the nucleolus. These results suggested that CSRVE1 was nucleoluslocalized transcription activator of proCsPME3. CsRVE1 (REVEILLE1) was an ortholog of AtRVE1 and belonged to a subfamily of Myb-like transcription factors that includes CIRCADIAN CLOCK-ASSOCIATED 1 (CCA1) and LATE ELONGATED HYPOCOTYL (LHY) clock components [53, 54]. RVE1 was reported to regulate the auxin biosynthesis, seed dormancy and germination, chlorophyll biosynthesis during plant development [53, $55,56]$. AtRVE1 acted as a negative regulator of freezing tolerance in Arabidopsis [57]. Here, we also found that the expression level of CsRVE1 was induced by low temperature stress. Thus, it could be concluded that CsRVE1 might be a low temperature-induced nucleolus-localized transcription activator of CSPME3. The cold weather in the deep winter induced the expression of transcription factor CsRVE1, then CsRVE1 binds to the promoter of $C S P M E 3$ and thus activated the expression of CsPME3. Subsequently, the activated pectin methylesterase activity leads to the modification of cell wall components, and thus resulted in the juice sac granulation of 'Lane late' navel orange fruits (Fig. 9).

\section{Conclusions}

In summary, a total of 53 CsPME genes were identified from Citrus sinensis, including 29 Type-I and 25 TypeII CsPMEs. Comprehensive bioinformatics analyses of phylogenetic relationship, gene structure, conserved 


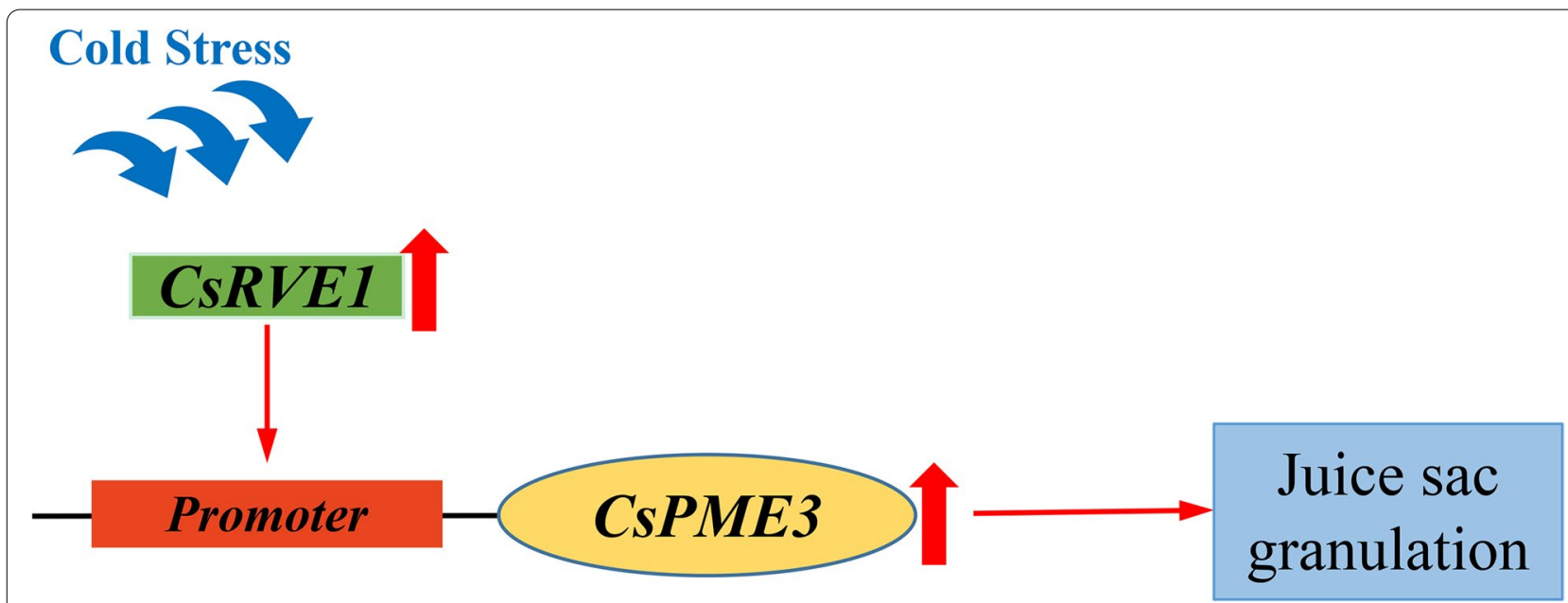

Fig. 9 Schematic diagram showing the cold-induced regulation pattern CSRVE1 on proCSPME3 involved in juice sac granulation of navel orange. The cold weather in the deep winter induces the expression of transcription factor CSRVE1, then CSRVE1 binds to the promoter of CSPME3 and thus activates the expression of CSPME3, which further leads to the juice sac granulation of 'Lane late' navel orange fruits

domains, chromosome localization, gene duplication, and synteny were performed on CsPME genes, which provide important clues for further investigation of CsPME genes. The expression profiles of CsPMEs and their subcellular localization analysis revealed that the CsPME genes were involved in the low temperatureinduced juice sac granulation in navel orange fruits. Moreover, the $\mathrm{Y} 1 \mathrm{H}$ and LUC assays revealed the regulatory effect of CsRVE1 on the activity of proCsPME3. Our results provide an insight into evolution, expression profiles, subcellular localization, and regulation patterns of the $P M E$ gene family in citrus. This study lays a solid foundation for further identification of the biological functions of CsPME gene during juice sac granulation of citrus.

\section{Methods}

\section{Identification of putative PME family genes from Citrus sinensis}

To identify CsPME genes, genome resources of sweet orange (Citrus sinensis, version 2) were downloaded from the Citrus Sinensis Annotation Project of Huazhong Agricultural University (http://citrus.hzau.edu.cn/ orange/) [40], and two BLAST methods were conducted. Firstly, the amino acid sequences of HMM (Hidden Markov Model, http://hmmer.janelia.org/) profile of the pectin Methyl-esterase (PME) domain (PF01095) were downloaded from Pfam database (http://pfam.xfam.org/, version 33.1) [58], and then used as queries for retrieving candidate $P M E$ family genes in $C$. sinensis genome using HMMER software (version 3.0, http://hmmer.org/) with an E-value cutoff of 1e-3 [59]. Secondly, the protein sequences of all known PME family genes in Arabidopsis thaliana were downloaded from TAIR database (http:// www.Arabidopsis.org/), and then utilized as queries for retrieving candidate $P M E$ family genes in $C$. sinensis genome by using BLAST-P with E-value cutoff of 1e-5. To ensure the presence of $P M E$ domain for each protein, all obtained candidate $P M E$ family genes were further confirmed by Simple Modular Architecture Research Tool (SMART, http://smart.embl-heidelberg.de/) and Conserved Domain Database (CCD, http://www.ncbi. nlm.nih.gov/cdd/) [60, 61]. Finally, the non-redundant and confirmed candidate genes were considered as citrus $P M E$ family genes, and the physicochemical properties of all CsPME proteins were predicted using ExPASy tool (http://web.expasy.org/protparam/) [62]. The gene loci of $P M E$ family genes were downloaded from Citrus Sinensis Annotation Project of Huazhong Agricultural University [40], and visualized using TBtools software [63]. The cis-elements of the promoter sequence of CsPME3 was analyzed using PlantCARE online tool (http://bioinforma tics.psb.ugent.be/webtools/plantcare/html/) according to the default parameters [64].

\section{Phylogenetic analysis, gene structure, and conserved motifs of $P M E$ family genes}

To explore the phylogenetic relationship of $P M E$ family genes between $C$. sinensis and A. thaliana, a multiple sequence alignment was performed using MAFFT (Multiple Alignment using Fast Fourier Transform, https://mafft.cbrc.jp/alignment/server/) with default parameters [65], and a phylogenetic tree was subsequently constructed using Maximum Likelihood Method of MEGA software with 1000 bootstraps based on the protein sequences of PMEs from $C$. sinensis and 
A.thaliana. [66]. The final phylogenetic tree was visualized and polished with the Interactive Tree of Life (iTOL, version 5, https://itol.embl.de/) [67]. The protein sequences of $A$. thaliana were downloaded from TAIR database (http://www.Arabidopsis.org/). The gene structures (exon-intron organizations) of $P M E$ family genes were presented using TBtools software. The conserved motifs were detected through the online tool of Multiple Em for Motif Elicitation program (MEME, version 5.1.1, http://meme-suite.org/tools/meme) using classic mode with the following parameters: (1) zero or one occurrence per sequence (zoops), (2) the optimum motif width ranging from 6 to 50 , (3) 15 as the maximum motif number $[68,69]$. Subsequently, the identified motifs were further annotated against an online database of InterPro (version 80.0, www.ebi.ac.uk/inter pro/search/sequence/) [70]. Finally, the combination figure of the phylogenetic tree, gene structures, and conserved motifs of $P M E$ family genes was generated using TBtools software [63].

\section{Gene duplication and synteny analysis}

The gene duplication of CsPME family genes was determined according to the criteria reported previously [71]. Two genes separated by less than 5 genes within $100 \mathrm{~kb}$ chromosome fragment were considered as tandemly duplicated genes [72]. The segmentally duplicated genes of CsPME family genes were identified with Multiple Collinearity Scan toolkit (MCScanX, [73]), and Circos software of TBtools was utilized for visualization [63]. MCScanX was further employed to analyze synteny relationship of the ortholog of $P M E$ family genes from C. sinensis, A. thaliana, and $O$. sativa with the default parameters. Synteny maps were generated by Dual Systeny Plotter software based on TBtools [63].

\section{Plant materials and treatments}

All plant materials used in this study were collected from mature 'Lane late' navel orange (Citrus sinensis Osbeck) trees in Zigui County of Hubei Province in the three gorgers reservoir area (E 110 41', N 30 $\left.{ }^{\circ} 54^{\prime}\right)$. Granulated 'Lane late' fruit samples were harvested at 350 DAF (days after flowering) in 2018 due to the extreme cold weather in the winter in reference to our previous study [37]. The average temperature in January of sampling site was -4 ${ }^{\circ} \mathrm{C}$ with an altitude of $520 \mathrm{~m}$, and the soil property was red yellow sandy soil with a pH value of 6.3. Each Fruit was halved by sterilized knife, and then divided into four granulation levels, namely, non-granulation (CK), slight granulation (GR1), moderate granulation (GR2), and serious granulation (GR3), according to a previous study [74]. Finally, the fruits samples at different granulation levels were collected. For low temperature treatment, the normal 'Lane late' fruits were placed on a plate and covered with sterilized gauze to keep moist. Then, the fruits were placed in an incubator at $4{ }^{\circ} \mathrm{C}$. The samples were collected at $0 \mathrm{~h}, 3 \mathrm{~h}, 6 \mathrm{~h}, 12 \mathrm{~h}, 24 \mathrm{~h}, 48 \mathrm{~h}$, and $72 \mathrm{~h}$. Each sample had three biological replicates with 3 individual fruits per biological replicate. All fruit samples were immediately frozen with liquid nitrogen and stored in $-80{ }^{\circ} \mathrm{C}$ for RNA extraction.

\section{Expression profiles of PME family genes in C. sinensis}

The transcriptome data of 4 fruit tissues (including epicarp, albedo, segment membrane, and juice sac) at 6 development stages (including 50 DAF, 80 DAF, 120 DAF, 155 DAF, 180 DAF, and 220 DAF) of 'Fengjie' navel orange from our previous study were utilized to investigate the spatial and temporal expression patterns of CsPME genes [41]. Specifically, the fruits were sampled in the second physiological fruit-falling period (50 day after flowering, DAF), in the expansion period (80, 120, and $155 \mathrm{DAF}$ ), in the coloring period (180 DAF), and in the full-ripening period (220 DAF). The transcriptional expression patterns of three independent bio-replicates were calculated by Fragments Per Kilobase per Million (FPKM). The heatmap was drawn using heatmap package of TBtools based on the $\log _{2}$-transformed FPKM values [63].

To identify the granulation-related CsPME genes, a transcriptome analysis was performed in 'Lane late' navel orange fruits at 4 different granulation levels, including non-granulation (CK), slight granulation (GR1), moderate granulation (GR2), and serious granulation (GR3). Total RNA was extracted from 12 'Lunwan' navel orange fruit samples (three biological replicates) using TRIzol ${ }^{\mathrm{TM}}$ reagent according to the manufacturer's instructions (Thermo Scientific). RNA quality was assessed by agarose gel electrophoresis and Agilent 2100 Bioanalyzer (Agilent Technologies), and then qualified RNA of all 12 samples were subjected to Berry Genomics (Beijing) for library construction and transcriptome sequencing. The gene expression level was assessed using HTSeq with FPKM method [75]. The differential expression analysis between pair of samples was performed using edgeR package [76], and genes with $\mid \log _{2}$ fold change $\mid \geq 1$ and p-adjusted value $\leq 0.05$ were considered as differentially expressed genes. Heatmap of differentially expressed CsPME genes was performed using $R$ package based on the $\log _{2}$ fold change values.

\section{RNA extraction, CDNA synthesis, and qRT-PCR of PME family genes in $C$. sinensis}

Total RNA was isolated from different samples using TRIzol $^{\circledR}$ Reagent (Invitrogen, USA) according to the manufacturer's instructions. RNA quality was assessed by 
agarose gel electrophoresis, and then one microgram of total RNA was used for ss/dsDNA digestion and cDNA synthesis with a $5 \mathrm{X}$ All-In-One RT MasterMix with AccuRT (Applied Biological Materials). Quantitative RTPCR was performed on 7500 Fast Real-Time PCR System (Applied Biosystems) with an EvaGreen 2X qPCR MasterMix (Applied Biological Materials). Elongation factor 1 (Ef1, Cs8g16990.1) and $\beta$-actin (Cs1g05000.1) were selected as internal references to reduce the systematic variance, and the relative abundance of $P M E$ genes was calculated with the average $C t$ values of $E f 1$ and $\beta$-actin using $2^{-\Delta \Delta \mathrm{Ct}}$ method [77]. The primers used for qRT-PCR were listed in Supplementary Table S5.

\section{Subcellular localization analysis}

The coding sequence (CDS) of $P M E$ family genes (PME4, PME8, PME30, PME51, PME54, PME55, PME76, and $P M E 95)$ without stop codon was cloned from 'Lane late' navel orange fruit CDNA, ligated with that of GFP module or mCherry module (exclusively for the genes localized on the apoplast), and cloned into pICH86988 vectors to construct 35S::PMEs:GFP, and 35S::PMEs:mCherry vectors through Golden Gate method [78]. The resultant vectors were transformed into Agrobacterium tumefaciens (strain GV3101 with pSoup-p19). The 35S::PMEs:GFP and 35S::PMEs:mCherry vectors together with corresponding markers were then co-expressed in tobacco leaves, including ER-marker (HDEL:OFP) [79], plasma membrane marker (CBL1n:OFP) [79], vacuole membrane marker (CBL3n:OFP) [79], and apoplast marker (RAmy3A:GFP) [80]. Additionally, the 35S::CsRVE1:GFP vector was constructed as described above and coexpressed with nucleolus marker (FIB2:mCherry) [43]. Transient expression experiment was performed by a previously-reported method [81]. The transformed tobacco plants were cultured in green house for 3 days, then the subcellular localization of $P M E$ family genes was observed and photographed via a confocal laser-scanning microscope (TCS SP6, Leica). The primers used for vector construction were listed in Supplementary Table S5.

\section{Yeast one-hybrid assay}

Yeast one-hybrid assay was conducted using the method reported by $\mathrm{Lu}$ et al. [82]. Specifically, the promoter sequence of $P M E 3$ was amplified from the genomic DNA extracted from 'Lane late' navel orange leaf. The promoter sequence was cloned into pAbAi vector to construct PME3-pAbAi bait vector. Subsequently, the obtained bait vector was transformed into Y1H Gold yeast, and the positive clones were selected with synthetic dextrose media lacking uracil (SD-Leu). Then, the positive bait was used to screen interactive transcription factors from a citrus Y1H library provided by Jihong Liu lab of Huazhong agricultural university. The pGADT7 empty vector (as a negative control) was transformed into yeast competent cell containing bait. The transformed yeast cells were diluted with a $10 \times$ dilution series and dotted onto SD-Leu plates with or without aureobasidin $\mathrm{A}(\mathrm{AbAi})$. If the cells grew on both types of media with or without $\mathrm{AbAi}$, the interaction of prey proteins and bait sequences was identified. The primers used for constructing $\mathrm{Y} 1 \mathrm{H}$ vectors were listed in Supplementary Table S5.

\section{Dual luciferase transcriptional activity assay}

The dual luciferase transcriptional activity (LUC) assay was performed according to a previously reported method [82]. To be more specific, the promoter sequence of PME3 was inserted into the upstream of LUC coding sequence in pGreen 0800-LUC to construct promoterLUC reporter vector. The CDS of CsRVE1 was cloned into AML4 over-expression vector to construct 35S::CsRVE1 effector vector. The effector and reporter vectors were transformed into Agrobacterium tumefaciens (strain GV3101 with pSoup-p19), and then co-expressed in tobacco leaves at an effector: reporter ratio of 1: 1 according to the method described by Hellens et al. [83]. After 3-day cultivation in green house, the LUC activity was determined using the Dual-Luciferase Reporter Assay System (Promega) with an infinite200 Pro microplate reader (Tecan). The primers used for constructing LUC vectors were listed in Supplementary Table S5.

\section{Abbreviations}

PME: Pectin methylesterase; Chr: Chromosome; CDS: Coding sequence; FPKM: Fragments per kilobase of transcript per million mapped reads; $Y 1 \mathrm{H}$ :Yeast one-hybrid assay; LUC: Dual luciferase transcriptional activity assay; GFP: Green fluorescent protein; OFP: Orange fluorescent protein; mCherry: mCherry red fluorescent protein; AbAi: Aureobasidin A.

\section{Supplementary Information}

The online version contains supplementary material available at https://doi. org/10.1186/s12864-022-08411-0.

Additional file 1: Figure S1. The expression patterns of four cell wall modification genes during juice sac granulation of navel orange.

Additional file 2: Figure $\mathbf{S 2}$. The predicted cis-elements of the promoter sequence of CSPME3.

Additional file 3: Table S1. Verification of PME and PMEl domains in candidate CSPME genes.

Additional file 4: Table S2. Basic information of the PME genes in Citrus sinensis.

Additional file 5: Table S3. The expression levels of CSPMEs in the transcriptome data of 4 fruit tissues at 6 development stages in 'Fengjie' navel orange. Four fruit tissues including albedo (AL), epicarp (EP), juice sac (JS), and segment membrane (SM), and six fruit development stages including 50 DAF (stage 1), 80 DAF (stage 2), 120 DAF (stage 3), 150 DAF (stage 4), $180 \mathrm{DAF}$ (stage 5), and $220 \mathrm{DAF}$ (stage 6). 
Additional file 6: Table S4. The expression levels of CSPMEs in the transcriptome data of 'Lane late' navel orange fruits under 4 different granulation levels, including non-granulation (CK), slight granulation (GR1), moderate granulation (GR2), and serious granulation (GR3).

Additional file 7: Table S5. List of primers used for qRT-PCR, gene cloning, and vector construction in this study.

\section{Acknowledgements}

Sincere gratitude goes to Professor Ping Liu from Huazhong Agriculture University, Wuhan, China for her English editing and language polishing of this manuscript.

\section{Authors' contributions}

FS and LW conceived this study; ZL and FS carried out the experiments and data analysis; YW, CW, GF, and FB had contributed in bioinformatic analysis; $L H, Z W$, and $X M$ participated in data analysis; $F S$ and $Z L$ written the draft of the manuscript; $J L$ and LW revised the manuscript; FS, LW, and YJ provided funding support. All authors have read and agreed to the published version of the manuscript.

\section{Funding}

This research was funded by the National Key Research and Development Program of China (2019YFD1001400), Key Research and Development Program of Hubei Province (2020BBA036), Hubei Provincial Agricultural Science and Technology Innovation Fund (2019-620-000-001-023), Hubei Provincial Natural Science Foundation of China (2020CFB452) and Youth Foundation of Hubei Academy of Agricultural Sciences (2020NKYJJ14).

\section{Availability of data and materials}

All Arabidopsis protein sequences were downloaded from The Arabidopsis Information Resource (TAIR) (https:// www.arabidopsis.org) all Citrus protein sequences were downloaded from the Citrus Sinensis Annotation Project (http:// citrus.hzau.edu.cn/orange/). The transcriptome data used in this study can be accessed with the Gene Expression Omnibus (GSE125726) from the NCBI database. The amplified coding sequences of CSPME3, CSPME6, CSPME21, CSPME34, CSPME35, CSPME40, CSPME52, and CSRVE1 can be accessed in the International Nucleotide Sequence Database Collaboration (INSDC) with the accession number of OM100720 OM100727, and the amplified promoter sequence of CSPME3 can be accessed with the accession number of OM100728 in INSDC.

\section{Declarations}

\section{Ethics approval and consent to participate}

The authors declare that permissions to collect all plants and their parts used in the study were obtained, and the collection of plant material complied with the institutional, national, and international guidelines and legislation.

\section{Consent for publication}

Not applicable.

\section{Competing interests}

The authors declare no competing interests.

\section{Author details}

${ }^{1}$ Institute of Fruit and Tea, Hubei Academy of Agricultural Sciences, Wuhan 430064, PR China. ${ }^{2}$ College of Horticulture and Forestry Sciences, Huazhong Agricultural University, Wuhan 430070, PR China.

Received: 16 August 2021 Accepted: 17 February 2022

Published online: 07 March 2022

\section{References}

1. Oh C-S, Kim H, Lee C. Rice cell wall polysaccharides: Structure and biosynthesis. J Plant Biol. 2013;56(5):274-82.

2. Zablackis E, Huang J, Muller B, Darvill AG, Albersheim P. Characterization of the cell-wall polysaccharides of Arabidopsis thaliana leaves. Plant Physiol. 1995;107(4):1129-38.
3. Anderson CT. We be jammin': an update on pectin biosynthesis, trafficking and dynamics. J Exp Bot. 2016;67(2):495-502.

4. Daher FB, Braybrook SA. How to let go: pectin and plant cell adhesion. Front Plant Sci. 2015;6:523.

5. Mohnen D. Pectin structure and biosynthesis. Curr Opin Plant Biol. 2008;11(3):266-77.

6. Philippe F, Pelloux J, Rayon C. Plant pectin acetylesterase structure and function: new insights from bioinformatic analysis. BMC Genomics. 2017;18(1):456

7. Wormit A, Usadel B. The Multifaceted Role of Pectin Methylesterase Inhibitors (PMEls). Int J Mol Sci. 2018;19(10):2878. https://doi.org/10. 3390/ijms19102878.

8. Levesque-Tremblay G, Muller K, Mansfield SD, Haughn GW. HIGHLY METHYL ESTERIFIED SEEDS is a pectin methyl esterase involved in embryo development. Plant Physiol. 2015;167(3):725-37.

9. Micheli F. Pectin methylesterases: cell wall enzymes with important roles in plant physiology. Trends Plant Sci. 2001;6(9):414-9.

10. Senechal F, Wattier C, Rusterucci C, Pelloux J. Homogalacturonanmodifying enzymes: structure, expression, and roles in plants. J Exp Bot. 2014;65(18):5125-60.

11. Pelloux J, Rusterucci C, Mellerowicz EJ. New insights into pectin methylesterase structure and function. Trends Plant Sci. 2007;12(6):267-77.

12. Rockel N, Wolf S, Kost B, Rausch T, Greiner S. Elaborate spatial patterning of cell-wall PME and PMEl at the pollen tube tip involves PMEI endocytosis, and reflects the distribution of esterified and de-esterified pectins. Plant J. 2008;53(1):133-43.

13. Jolie RP, Duvetter T, Van Loey AM, Hendrickx ME. Pectin methylesterase and its proteinaceous inhibitor: a review. Carbohydr Res. 2010;345(18):2583-95.

14. Louvet R, Cavel E, Gutierrez L, Guenin S, Roger D, Gillet F, Guerineau F, Pelloux J. Comprehensive expression profiling of the pectin methylesterase gene family during silique development in Arabidopsis thaliana. Planta. 2006;224(4):782-91.

15. Duan W, Huang Z, Song X, Liu T, Liu H, Hou X, Li Y. Comprehensive analysis of the polygalacturonase and pectin methylesterase genes in Brassica rapa shed light on their different evolutionary patterns. Sci Rep. 2016:6:25107.

16. Li W, Shang H, Ge Q, Zou C, Cai J, Wang D, Fan S, Zhang Z, Deng X, Tan Y. Genome-wide identification, phylogeny, and expression analysis of pectin methylesterases reveal their major role in cotton fiber development. BMC Genomics. 2016;17(1):1-13.

17. Jeong HY, Nguyen HP, Lee C. Genome-wide identification and expression analysis of rice pectin methylesterases: Implication of functional roles of pectin modification in rice physiology. J Plant Physiol. 2015;183:23-9.

18. Pinzon-Latorre D, Deyholos MK. Characterization and transcript profiling of the pectin methylesterase (PME) and pectin methylesterase inhibitor (PMEI) gene families in flax (Linum usitatissimum). BMC Genomics. 2013;14:742.

19. Wen B, Zhang F, Wu X, Li H. Characterization of the Tomato (Solanum lycopersicum) Pectin Methylesterases: Evolution, Activity of Isoforms and Expression During Fruit Ripening. Front Plant Sci. 2020;11:238.

20. Kagan-Zur V, Tieman DM, Marlow SJ, Handa AK. Differential regulation of polygalacturonase and pectin methylesterase gene expression during and after heat stress in ripening tomato (Lycopersicon esculentum Mill.) fruits. Plant Mol Biol. 1995;29(6):1101-10.

21. Wen B, Strom A, Tasker A, West G, Tucker GA. Effect of silencing the two major tomato fruit pectin methylesterase isoforms on cell wall pectin metabolism. Plant Biol (Stuttg). 2013;15(6):1025-32.

22. Phan TD, Bo W, West G, Lycett GW, Tucker GA. Silencing of the major salt-dependent isoform of pectinesterase in tomato alters fruit softening. Plant Physiol. 2007;144(4):1960-7.

23. Bosch M, Hepler PK. Pectin methylesterases and pectin dynamics in pollen tubes. Plant Cell. 2005;17(12):3219-26.

24. Jiang L, Yang SL, Xie LF, Puah CS, Zhang XQ, Yang WC, Sundaresan V, Ye D. VANGUARD1 encodes a pectin methylesterase that enhances pollen tube growth in the Arabidopsis style and transmitting tract. Plant Cell. 2005;17(2):584-96.

25. Guenin S, Hardouin J, Paynel F, Muller K, Mongelard G, Driouich A, Lerouge P, Kermode AR, Lehner A, Mollet JC, et al. AtPME3, a ubiquitous cell wall pectin methylesterase of Arabidopsis thaliana, alters the 
metabolism of cruciferin seed storage proteins during post-germinative growth of seedlings. J Exp Bot. 2017;68(5):1083-95.

26. Bethke G, Grundman RE, Sreekanta S, Truman W, Katagiri F, Glazebrook J. Arabidopsis PECTIN METHYLESTERASEs contribute to immunity against Pseudomonas syringae. Plant Physiol. 2014;164(2):1093-107.

27. Giancaspro A, Lionetti V, Giove SL, Zito D, Fabri E, Reem N, Zabotina OA, De Angelis E, Monaci L, Bellincampi $D$, et al. Cell wall features transferred from common into durum wheat to improve Fusarium Head Blight resistance. Plant Sci. 2018;274:121-8.

28. Yan J, He H, Fang L, Zhang A. Pectin methylesterase31 positively regulates salt stress tolerance in Arabidopsis. Biochem Biophys Res Commun. 2018;496(2):497-501.

29. Wu H-C, Huang Y-C, Stracovsky L, Jinn T-L. Pectin methylesterase is required for guard cell function in response to heat. Plant Signal Behav. 2017;12(6):e1338227

30. Zhang H, Xie Y, Liu C, Chen S, Hu S, Xie Z, Deng X, Xu J. Comprehensive comparative analysis of volatile compounds in citrus fruits of different species. Food Chem. 2017;230:316-26.

31. Chen J, Yuan Z, Zhang H, Li W, Shi M, Peng Z, Li M, Tian J, Deng X, Cheng $Y$, et al. Cit 1,2RhaT and two novel CitdGlcTs participate in flavorrelated flavonoid metabolism during citrus fruit development. J Exp Bot. 2019;70(10):2759-71.

32. Wang S, Yang C, Tu H, Zhou J, Liu X, Cheng Y, Luo J, Deng X, Zhang H, $\mathrm{Xu}$ J. Characterization and Metabolic Diversity of Flavonoids in Citrus Species. Sci Rep. 2017;7(1):10549.

33. Xu CJ, Fraser PD, Wang WJ, Bramley PM. Differences in the carotenoid content of ordinary citrus and lycopene-accumulating mutants. J Agric Food Chem. 2006;54(15):5474-81.

34. Zhu F, Luo T, Liu C, Wang Y, Yang H, Yang W, Zheng L, Xiao X, Zhang M, Xu $R$, et al. An R2R3-MYB transcription factor represses the transformation of alpha- and beta-branch carotenoids by negatively regulating expression of $\mathrm{CrBCH} 2$ and $\mathrm{CrNCED} 5$ in flavedo of Citrus reticulate. New Phytol. 2017:216(1):178-92

35. Han HB, Li H, Hao RL, Chen YF, Ni H, Li HH. One-step column chromatographic extraction with gradient elution followed by automatic separation of volatiles, flavonoids and polysaccharides from Citrus grandis. Food Chem. 2014;145:542-8.

36. Jia N, Liu J, Tan P, Sun Y, Lv Y, Liu J, Sun J, Huang Y, Lu J, Jin N, et al. Citrus sinensis MYB Transcription Factor CsMYB85 Induce Fruit Juice Sac Lignification Through Interaction With Other CsMYB Transcription Factors. Front Plant Sci. 2019;10:213.

37. Wu LM, Wang C, He LG, Wang ZJ, Tong Z, Song F, Tu JF, Qiu WM, Liu JH, Jiang YC et al: Transcriptome Analysis Unravels Metabolic and Molecular Pathways Related to Fruit Sac Granulation in a Late-Ripening Navel Orange (Citrus sinensis Osbeck). Plants (Basel) 2020, 9(1).

38. Wu JL, Pan TF, Guo ZX, Pan DM. Specific lignin accumulation in granulated juice sacs of Citrus maxima. J Agric Food Chem. 2014;62(50):12082-9.

39. Yao S, Cao Q, Xie J, Deng L, Zeng K. Alteration of sugar and organic acid metabolism in postharvest granulation of Ponkan fruit revealed by transcriptome profiling. Postharvest Biol Technol. 2018;139:2-11.

40. Xu Q, Chen LL, Ruan X, Chen D, Zhu A, Chen C, Bertrand D, Jiao WB, Hao $\mathrm{BH}$, Lyon MP, et al. The draft genome of sweet orange (Citrus sinensis). Nat Genet. 2013:45(1):59-66.

41. Feng G, Wu J, Xu Y, Lu L, Yi H. High-spatiotemporal-resolution transcriptomes provide insights into fruit development and ripening in Citrus sinensis. Plant Biotechnol J. 2021;19(7):1337-53. https://doi.org/10.1111/ pbi.13549.

42. Batoko H, Zheng HQ, Hawes C, Moore I. A rab1 GTPase is required for transport between the endoplasmic reticulum and golgi apparatus and for normal golgi movement in plants. Plant Cell. 2000;12(11):2201-18.

43. Chang C-H, Hsu F-C, Lee S-C, Lo Y-S, Wang J-D, Shaw J, Taliansky M, Chang B-Y, Hsu Y-H, Lin N-S. The nucleolar fibrillarin protein is required for helper virus-independent long-distance trafficking of a subviral satellite RNA in plants. Plant Cell. 2016;28(10):2586-602.

44. Guenin S, Mareck A, Rayon C, Lamour R, AssoumouNdong Y, Domon $J M$, Senechal F, Fournet F, Jamet E, Canut H, et al. Identification of pectin methylesterase 3 as a basic pectin methylesterase isoform involved in adventitious rooting in Arabidopsis thaliana. New Phytol. 2011;192(1):114-26.

45. Hewezi T, Howe P, Maier TR, Hussey RS, Mitchum MG, Davis EL, Baum TJ. Cellulose binding protein from the parasitic nematode Heterodera schachtii interacts with Arabidopsis pectin methylesterase: cooperative cell wall modification during parasitism. Plant Cell. 2008;20(11):3080-93.

46. Weber M, Deinlein U, Fischer S, Rogowski M, Geimer S, Tenhaken R, Clemens S. A mutation in the Arabidopsis thaliana cell wall biosynthesis gene pectin methylesterase 3 as well as its aberrant expression cause hypersensitivity specifically to Zn. Plant J. 2013;76(1):151-64.

47. Raiola A, Lionetti V, Elmaghraby I, Immerzeel P, Mellerowicz EJ, Salvi G, Cervone F, Bellincampi D. Pectin methylesterase is induced in Arabidopsis upon infection and is necessary for a successful colonization by necrotrophic pathogens. Mol Plant Microbe Interact. 2011;24(4):432-40.

48. Yao S, Wang Z, Cao Q, Xie J, Wang X, Zhang R, Deng L, Ming J, Zeng K. Molecular basis of postharvest granulation in orange fruit revealed by metabolite, transcriptome and methylome profiling. Postharvest Biol Technol. 2020;166:111205.

49. Solecka D, Zebrowski J, Kacperska A. Are pectins involved in cold acclimation and de-acclimation of winter oil-seed rape plants? Ann Bot. 2008;101(4):521-30.

50. Qu T, Liu R, Wang W, An L, Chen T, Liu G, Zhao Z. Brassinosteroids regulate pectin methylesterase activity and AtPME41 expression in Arabidopsis under chilling stress. Cryobiology. 2011;63(2):111-7.

51. Lee JY, Lee DH. Use of serial analysis of gene expression technology to reveal changes in gene expression in Arabidopsis pollen undergoing cold stress. Plant Physiol. 2003;132(2):517-29.

52. Senechal F, L'Enfant M, Domon JM, Rosiau E, Crepeau MJ, Surcouf O, Esquivel-Rodriguez J, Marcelo P, Mareck A, Guerineau F, et al. Tuning of Pectin Methylesterification: pectin methylesterase inhibitor 7 modulates the processive activity of co-expressed pectin methylesterase 3 in a pHdependent manner. J Biol Chem. 2015;290(38):23320-35.

53. Rawat R, Schwartz J, Jones MA, Sairanen I, Cheng Y, Andersson CR, Zhao Y, Ljung K, Harmer S. REVEILLE1, a Myb-like transcription factor, integrates the circadian clock and auxin pathways. Proc Natl Acad Sci USA. 2009;106(39):16883-8.

54. Rawat R, Takahashi N, Hsu PY, Jones MA, Schwartz J, Salemi MR, Phinney BS, Harmer S. REVEILLE8 and PSEUDO-REPONSE REGULATOR5 form a negative feedback loop within the Arabidopsis circadian clock. Plos Genet. 2011;7(3):e1001350.

55. Xu G, Guo H, Zhang D, Chen D, Jiang Z, Lin R. REVEILLE1 promotes NADPH: protochlorophyllide oxidoreductase A expression and seedling greening in Arabidopsis. Photosynth Res. 2015;126(2):331-40.

56. Jiang Z, Xu G, Jing Y, Tang W, Lin R. Phytochrome B and REVEILLE1/2mediated signalling controls seed dormancy and germination in Arabidopsis. Nat Commun. 2016;7(1):1-10.

57. Meissner M, Orsini E, Ruschhaupt M, Melchinger AE, Hincha DK, Heyer AG. Mapping quantitative trait loci for freezing tolerance in a recombinant inbred line population of a Rabidopsis thaliana accessions tenela and C24 reveals reveille1 as negative regulator of cold acclimation. Plant Cell Environ. 2013;36(7):1256-67.

58. El-Gebali S, Mistry J, Bateman A, Eddy SR, Luciani A, Potter SC, Qureshi M, Richardson LJ, Salazar GA, Smart A, et al. The Pfam protein families database in 2019. Nucleic Acids Res. 2019;47(D1):D427-32.

59. Potter SC, Luciani A, Eddy SR, Park Y, Lopez R, Finn RD. HMMER web server: 2018 update. Nucleic Acids Res. 2018;46(W1):W200-4.

60. Letunic I, Bork P. 20 years of the SMART protein domain annotation resource. Nucleic Acids Res. 2018;46(D1):D493-6.

61. Lu S, Wang J, Chitsaz F, Derbyshire MK, Geer RC, Gonzales NR, Gwadz M, Hurwitz DI, Marchler GH, Song JS, et al. CDD/SPARCLE: the conserved domain database in 2020. Nucleic Acids Res. 2020;48(D1):D265-8.

62. Gasteiger E, Gattiker A, Hoogland C, Ivanyi I, Appel RD, Bairoch A. ExPASy: The proteomics server for in-depth protein knowledge and analysis. Nucleic Acids Res. 2003;31(13):3784-8.

63. Chen C, Chen H, Zhang Y, Thomas HR, Frank MH, He Y, Xia R. TBtools: An Integrative Toolkit Developed for Interactive Analyses of Big Biological Data. Mol Plant. 2020;13(8):1 194-202. https://doi.org/10.1016/j.molp.2020. 06.009 .

64. Lescot M, Dehais P, Thijs G, Marchal K, Moreau Y, Van de Peer Y, Rouze P, Rombauts S. PlantCARE, a database of plant cis-acting regulatory elements and a portal to tools for in silico analysis of promoter sequences. Nucleic Acids Res. 2002;30(1):325-7.

65. Katoh K, Rozewicki J, Yamada KD. MAFFT online service: multiple sequence alignment, interactive sequence choice and visualization. Brief Bioinform. 2019;20(4):1160-6. 
66. Kumar S, Stecher G, Li M, Knyaz C, Tamura K. MEGA X: Molecular Evolutionary Genetics Analysis across Computing Platforms. Mol Biol Evol. 2018;35(6):1547-9.

67. Letunic I, Bork P. Interactive tree of life (iTOL) v3: an online tool for the display and annotation of phylogenetic and other trees. Nucleic Acids Res. 2016;44(W1):W242-245.

68. Bailey TL, Boden M, Buske FA, Frith M, Grant CE, Clementi L, Ren J, Li WW, Noble WS. MEME SUITE: tools for motif discovery and searching. Nucleic Acids Res. 2009;37(Web Server issue):W202-208.

69. Bailey TL, Elkan C. Fitting a mixture model by expectation maximization to discover motifs in biopolymers. Proc Int Conf Intell Syst Mol Biol. 1994;2:28-36.

70. Mitchell AL, Attwood TK, Babbitt PC, Blum M, Bork P, Bridge A, Brown SD, Chang HY, El-Gebali S, Fraser MI, et al. InterPro in 2019: improving coverage, classification and access to protein sequence annotations. Nucleic Acids Res. 2019;47(D1):D351-60.

71. Yang S, Zhang X, Yue JX, Tian D, Chen JQ. Recent duplications dominate NBS-encoding gene expansion in two woody species. Mol Genet Genomics. 2008;280(3):187-98.

72. Wang L, Guo K, Li Y, Tu Y, Hu H, Wang B, Cui X, Peng L. Expression profiling and integrative analysis of the CESA/CSL superfamily in rice. BMC Plant Biol. 2010;10:282.

73. Wang Y, Tang H, Debarry JD, Tan X, Li J, Wang X, Lee TH, Jin H, Marler B, Guo H, et al. MCScanX: a toolkit for detection and evolutionary analysis of gene synteny and collinearity. Nucleic Acids Res. 2012;40(7):e49.

74. Zhang J, Wang M, Cheng F, Dai C, Sun Y, Lu J, Huang Y, Li M, He Y, Wang $F$, et al. Identification of microRNAs correlated with citrus granulation based on bioinformatics and molecular biology analysis. Postharvest Biol Technol. 2016;118:59-67.

75. Trapnell C, Williams BA, Pertea G, Mortazavi A, Kwan G, van Baren MJ, Salzberg SL, Wold BJ, Pachter L. Transcript assembly and quantification by RNA-Seq reveals unannotated transcripts and isoform switching during cell differentiation. Nat Biotechnol. 2010;28(5):511-5.

76. Robinson MD, McCarthy DJ, Smyth GK. edgeR: a Bioconductor package for differential expression analysis of digital gene expression data. Bioinformatics. 2010;26(1):139-40.

77. Livak KJ, Schmittgen TD. Analysis of relative gene expression data using real-time quantitative PCR and the 2(-Delta Delta C(T)) Method. Methods. 2001;25(4):402-8.

78. Engler C, Youles M, Gruetzner R, Ehnert TM, Werner S, Jones JD, Patron $\mathrm{NJ}$, Marillonnet S. A golden gate modular cloning toolbox for plants. ACS Synth Biol. 2014;3(11):839-43.

79. Batistic O, Sorek N, Schultke S, Yalovsky S, Kudla J. Dual fatty acyl modification determines the localization and plasma membrane targeting of CBL/CIPK Ca2+ signaling complexes in Arabidopsis. Plant Cell. 2008;20(5):1346-62.

80. Chen MH, Liu LF, Chen YR, Wu HK, Yu SM. Expression of a-amylases, carbohydrate metabolism, and autophagy in cultured rice cells is coordinately regulated by sugar nutrient. Plant J. 1994;6(5):625-36.

81. Sparkes IA, Runions J, Kearns A, Hawes C. Rapid, transient expression of fluorescent fusion proteins in tobacco plants and generation of stably transformed plants. Nat Protoc. 2006; 1(4):2019-25.

82. Lu S, Zhang Y, Zhu K, Yang W, Ye J, Chai L, Xu Q, Deng X. The Citrus Transcription Factor CsMADS6 Modulates Carotenoid Metabolism by Directly Regulating Carotenogenic Genes. Plant Physiol. 2018;176(4):2657-76.

83. Hellens RP, Allan AC, Friel EN, Bolitho K, Grafton K, Templeton MD, Karunairetnam S, Gleave AP, Laing WA. Transient expression vectors for functional genomics, quantification of promoter activity and RNA silencing in plants. Plant Methods. 2005;1:13.

\section{Publisher's Note}

Springer Nature remains neutral with regard to jurisdictional claims in published maps and institutional affiliations. 